\title{
Analysis and Simulation of Queueing Models for Reservation Based Bandwidth Access with Large Propagation Delays
}

\author{
S. Gouri Sankar and Anurag Kumar \\ Dept. of Electrical Communication Engineering \\ Indian Institute of Science, Bangalore, 560 012, INDIA
}

\begin{abstract}
Motivated by reservation based multiple access protocols in satellite networks, in this paper we consider discrete time queueing models in which the allocation of a server to the queue/s is based on delayed queue length information. Service allocations are made at frame boundaries, and in general a frame consists of several slots, each of which can carry one packet. We analyse a model in which the service allocation at each frame boundary is based on the most recently observed queue length. In this model the queue length process is a Markov chain of order $\Delta+1$, where $\Delta$ is the round trip delay in frame times. An interesting aspect of this model is that bandwidth allocations go waste, since the queue to which bandwidth is allocated may not have that many packets. For the case of a single node, independent and identically distributed arrivals, and when each frame has one slot, we are able to obtain explicit expressions for the generating function of the stationary queue length. A decomposition formula similar that found in vacation models is shown to hold. We use simulations to study the case of multiple slots per frame, and the case of two nodes. We also examine a model in which the bandwidth scheduler bases its allocations on the most recently observed queue length, and on the past allocations already made. We show how the mean queue length, the coefficient of variation of queue length, and the average wasted reservations vary with arrival rate, and the round trip delay $\Delta$.
\end{abstract}

\section{INTRODUCTION}

It is well known that the performance of contention based multiple access protocols deteriorates with increasing link speeds and propagation delays. Hence in high-speed networks that span large distances (e.g., metropolitan area optical networks, or satellite networks) conflict free multiple access protocols are used. Further, among conflict free access protocols, for packet switched transport, and where bandwidth reservation and quality-of-service guarantees are required, reservation based multiple access protocols are the most effective (see [6]). In such situations, the nodes contending for the shared bandwidth send reservation requests to a central scheduler, which considers all the requests and the available bandwidth, and makes explicit allocations to each of the requesting nodes ([2], [5], [8]. Owing to large propagation delays (often several frames (see [3])) in such networks, the central scheduler has to work with "stale" information, and there is several frames of delay between a node making a request and being allocated bandwidth.

In this paper we consider queueing models that arise from reservation bandwidth access with large propagation delays.

S. Gouri Sankar is currently with Silicon Automation Systems (India) Ltd., Bangalore, INDIA.

Email address for correspondence: anurag@ece.iisc.ernet.in
The link is organised into periodic frames, each with an equal number of slots, each of which can accommodate one packet. Such would exactly be the case for a satellite ATM link ([2]). The round trip time is taken to be $\Delta$ frame times, where $\Delta \in\{0,1,2, \ldots\}$. We first consider a single node, and assume that, in each frame, the bandwidth reservation scheduler allots bandwidth to this single queue based on the most recently observed queue length. For independent and identically distributed (i.i.d.) arrivals in each frame, the queue length at the node is a $(\Delta+1)$ th order Markov chain. We work with the $(\Delta+1)$ th order moment generating function for this Markov model. We carry out this analysis, and present explicit formulas for some cases. A decomposition formula similar to that found in vacation models is shown to hold. The model assumes that the scheduler bases its allocation on the most recently observed queue length at the scheduler; i.e., it does not take into account the allocations already made. An important issue in this scheme (Scheme 1) is that, since the bandwidth allocation is based on delayed information, bandwidth allocation in a frame may actually not get utilised, since by then the queue has emptied out. For comparison, we present simulation results for another scheme (Scheme 2 [1]) in which the scheduler also accounts for the reservations already made; the scheduler reserves bandwidth in a frame only if it is absolutely sure, given the previous allocations, and delayed queue lengths, that the allocated bandwidth will not go waste.

We study the effect of varying the frame size, for a fixed propagation delay. Based on our analysis and simulation, we find that it is better to use shorter frames; this is to be expected, since with shorter frames, the scheduler gets more frequent feedbacks.

For more than one node, the exact analytical approach is hard even without the complication of propagation delays. In this paper we only present simulation results, for the two queue model with propagation delays. Also here we only consider Bernoulli arrivals. An approximate analytical approach for more than one queue, and simulation results for more realistic traffic (on-off variable bit rate, and window flow controlled (as in TCP)) are presented in [4].

\section{Queueing Models With Service Reservation DELAYS}

Reservation requests for slots in future frames are made by nodes at the beginning of each frame. We do not con- 
sider the problem of multiple access to the reservation request slots, and assume that each node can send reservation control information in every frame ([8]). There is a central reservation scheduler that allocates reserved slots to the transmitter on the uplink. We can generalize the notion of a "reservation" being requested by a node to "information" being sent by the node (e.g., current queue length), on the basis of which the reservation scheduler can decide how much bandwidth to reserve. The delay between the epoch that a reservation request is transmitted to the reservation scheduler, and the reservation allocation is received by the node is taken to be an integral number of frames denoted by $\Delta=0,1,2, \ldots$.

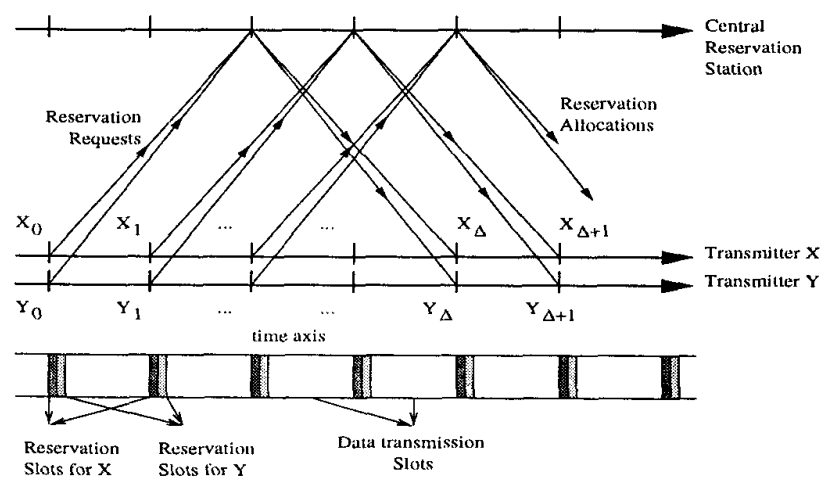

Fig. 1. Two nodes make reservation requests in each frame, and there is a reservation delay of $\Delta$ data frames; shown is the case of $\Delta=4$.

In Figure 1 we show the interaction between the central reservation scheduler and two queues, whose queue lengths are denoted by $X$ and $Y$. The diagram assumes that the delays are the same for both the transmitters. $X_{k}$ and $Y_{k}$ represent the queue lengths at the transmitters at the beginning of frame $k$. Observe that a reservation request (or queue length information) sent by the transmitters at the beginning of frame $k$, arrives at the scheduler after $\Delta / 2$ frames, and the resulting bandwidth allocation reaches the nodes at the beginning of frame $k+\Delta$. We can thus view the model as if at the beginning of each frame the number of slots alloted to each queue depends on at least $\Delta$ frames old queue history.

The following notation and assumptions hold throughout the paper: each frame has a service capacity of $c$ slots; reservations are only alloted at frame boundaries, and any arrivals in a frame only get served in the next frame or later; at each node the number of packet arrivals in the frames are i.i.d. random variables, $\left\{A_{k}\right\}$, with $\operatorname{Prob}\left(A_{k}=i\right)=a_{i}, 0 \leq$ $i<\infty$; let the generator function of this probability mass function be represented by $\tilde{a}(u)$, i.e., $\tilde{a}(u)=\sum_{i=0}^{\infty} a_{i} u^{i}$.

We consider two schemes that the scheduler could use to allocate bandwidth:

Scheme 1: The allocation in slot $k$ is based on the queue length information upto slot $k-\Delta$. The scheduler does not account for the allocations that it has already made; thus there is a possibility of allocating bandwidth to an empty queue. Scheme 1 can be taken to correspond to the situation in which $\Delta$ is not known at the scheduler, and a naive allocation based on observed queue lengths is made. Scheme 2: Bandwidth allocation in slot $k$ is made based on subtracting the total amount of bandwidth allocation made to a queue during the previous round-trip delay ( $\Delta$ slots) from the known queue length for the queue. Obviously in this scheme bandwidth is never wasted, but bandwidth may not be allocated even if new packets have arrived during the delay period.

For the case of a single node, we provide an analysis of Scheme 1, and evaluate some expressions for $c=1$ and small $\Delta$. Scheme 2 is analysed in [1]. Simulation results are used to study and compare the two schemes for larger frame capacities and delays, and for the two queue case.

\section{Single Node AnAlysis}

\section{A. The General Model}

The frame boundaries are indexed by $k, k=0,1,2, \ldots$; by the $k$ th frame we mean the frame whose boundaries are indexed by $k$ and $k+1$. Consider a single node, and let $X_{k}$ denote the queue length at the beginning of frame $k$. The following is a natural generalisation of the usual evolution equation to the case $\Delta>0$ :

$$
X_{k+1}=\left(X_{k}-\min \left(X_{k-\Delta}, c\right)\right)^{+}+A_{k+1}
$$

It is clear that $\left\{X_{k}\right\}$ is a $\Delta+1$ order Markov chain. Consider the $\Delta+1$ order generator function

$$
\begin{aligned}
& \tilde{g}_{\Delta}\left(u_{0}, \ldots, u_{\Delta}\right)=E\left[u_{0}^{X_{0}} \ldots u_{\Delta}^{X}\right] \\
& \quad=\sum_{i_{0}, \ldots, i_{\Delta}} p_{0 \Delta}\left(i_{0}, i_{1}, \ldots, i_{\Delta}\right) u_{0}^{i_{0}} \cdot \ldots \cdot u_{\Delta}^{i_{\Delta}}
\end{aligned}
$$

where $p_{0 \Delta}\left(i_{0}, i_{1}, \ldots, i_{\Delta}\right)$ is the joint probability distribution for $\left(X_{0}, \ldots, X_{\Delta}\right)$. Similarly, define

$$
\tilde{g}_{\Delta+1}\left(u_{1}, \ldots, u_{\Delta+1}\right)=E\left[u_{1}^{X_{1}} \ldots u_{\Delta+1}^{X_{\Delta+1}}\right]
$$

B. Single Slot per Frame: $c=1, \Delta \geq 1$

With $c=1$, conditioning on $\left(X_{0}, \ldots, X_{\Delta}\right)$, it is easy to obtain

$$
\begin{aligned}
& \tilde{g}_{\Delta+1}\left(u_{1}, \ldots, u_{\Delta+1}\right)= \\
& \quad \tilde{a}\left(u_{\Delta+1}\right)\left\{( 1 - \frac { 1 } { u _ { \Delta + 1 } } ) \left[\tilde{g}_{\Delta}\left(1, u_{1}, \ldots, u_{\Delta-1}, 0\right)\right.\right. \\
& +\tilde{g}_{\Delta}\left(0, u_{1}, \ldots, u_{\Delta-1}, u_{\Delta} u_{\Delta+1}\right) \\
& \left.\quad-\tilde{g}_{\Delta}\left(0, u_{1}, \ldots, u_{\Delta-1}, 0\right)\right] \\
& \left.+\frac{\tilde{g}_{\Delta}\left(1, u_{1}, \ldots, u_{\Delta-1}, u_{\Delta} u_{\Delta+1}\right)}{u_{\Delta+1}}\right\}
\end{aligned}
$$

To obtain the stationary probability distribution we take $\tilde{g}_{\Delta+1}=\tilde{g}_{\Delta}=\tilde{g}$ in $(5)$, yielding

$$
\begin{aligned}
& \tilde{g}\left(u_{1}, \ldots, u_{\Delta+1}\right)= \\
& \quad \tilde{a}\left(u_{\Delta+1}\right)\left\{( 1 - \frac { 1 } { u _ { \dot { \Delta } + 1 } } ) \left[\tilde{g}\left(1, u_{1}, \ldots, u_{\Delta-1}, 0\right)\right.\right.
\end{aligned}
$$




$$
\left.\begin{array}{l}
+\tilde{g}\left(0, u_{1}, \ldots, u_{\Delta-1}, u_{\Delta} u_{\Delta+1}\right) \\
\left.-\tilde{g}\left(0, u_{1}, \ldots, u_{\Delta-1}, 0\right)\right] \\
+\frac{\tilde{g}\left(1, u_{1}, \ldots, u_{\Delta-1}, u_{\Delta} u_{\Delta+1}\right)}{u_{\Delta+1}}
\end{array}\right\}
$$

Setting $u_{1}=u_{2}=\ldots=u_{\Delta}=1$ and $u_{\Delta+1}=u$ in Equation 6, we obtain the marginal generating function $\tilde{g}(1,1, \cdots, 1, u)$. Setting $\lim _{u \rightarrow 1} \tilde{g}(1,1, \cdots, 1, u)=1$ (i.e., the invariant vector is summable), and using L'Hospital's rule, we get

$$
1-\tilde{a}^{\prime}(1)=\tilde{g}(1, \ldots, 1,0)+\tilde{g}(0,1, \ldots, 1)-\tilde{g}(0,1, \ldots, 1,0)
$$

We have $E A=\tilde{a}^{\prime}(1)$, and for stationary $\left\{X_{k}\right\}$ $\tilde{g}(1, \ldots, 1,0)=\tilde{g}(0,1, \ldots, 1)=\operatorname{Prob}\left(X_{k}=0\right)$ for all $k$; also $\tilde{g}(0,1, \ldots, 1,0)=\operatorname{Prob}\left(X_{k-\Delta}=0, X_{k}=0\right)$. Observing that $\operatorname{Prob}\left(X_{k}=0 \mid X_{k-\Delta}=0\right)=\frac{\tilde{g}(0,1, \ldots, 1,0)}{\tilde{g}(0,1, \ldots, 1)}$, we define this conditional probability to be $f(\tilde{a})$; i.e.,

$$
\tilde{g}(0,1, \ldots, 1,0)=f(\tilde{a}) \cdot \tilde{g}(1, \ldots, 1,0)
$$

Then from (7) we can write

$$
\tilde{g}(1, \ldots, 1,0)=\tilde{g}(0,1, \ldots, 1)=\frac{1-E A}{2-f(\tilde{a})}
$$

Thus, denoting by $\Pi_{j}, j \geq 0$, the stationary marginal distribution of the process $\left\{X_{k}\right\}$, we have $\Pi_{0}=\frac{1-E A}{2-f(\tilde{a})}$. Since $0 \leq f(\tilde{a}) \leq 1$, it follows that $\Pi_{0}>0$ iff $E A<1$. It follows that the $(\Delta+1)$ order Markov chain is positive recurrent iff $E A<1$, as might have been expected. Also we have the marginal generating function

$$
\begin{aligned}
& \tilde{g}(1, \ldots, 1, u)=\frac{(1-E A) \cdot \tilde{a}(u) \cdot(1-u)}{\tilde{a}(u)-u} \\
& {\left[\frac{\tilde{g}(1, \ldots, 1,0)(1-f(\tilde{a}))+\tilde{g}(0,1, \ldots, u)}{1-E A}\right] }
\end{aligned}
$$

Thus for $X$ representing the stationary random variable, $E\left[u^{X}\right]$ is given by the right hand side of (10). It is easy to see that for zero information delay the generating function for the queue length is $\frac{(1-E A) \cdot \vec{a}(u) \cdot(1-u)}{\bar{a}(u)-u}$. Thus, with information delay the generating function is the product of two terms: the generating function without delay, and another generating function that depends on the arrival distribution and $\Delta$. Such decomposition results are well known in vacation models, and in the context of queues with server allocation based on delayed information another such result has been reported in [1].

Mean queue length: We have

$$
E X=\lim _{u \rightarrow 1} \frac{d}{d u} \tilde{g}(1, \ldots, 1, u)
$$

Defining $\tilde{g}(0,1, \ldots, 1, \dot{\mathrm{i}})=\left(\frac{\partial}{\partial u} \tilde{g}(0,1, \ldots, 1, u)\right)_{u=1}$, we find that

$$
\begin{aligned}
E X & =\frac{\tilde{g}(0,1, \ldots, 1, \dot{1})}{1-E A} \\
& +\tilde{g}(1, \ldots, 1,0)(2-f(\tilde{a})) \frac{\left[E A^{2}-2 . E^{2} A+E A\right]}{2(1-E A)^{2}}(11)
\end{aligned}
$$

Define $\tilde{g}(0,1, \ldots, 1, \dot{1})=h(\tilde{a}) \tilde{g}(1, \ldots, 1,0)$, and observe that $h(\tilde{a})$ is just a conditional expectation:

$$
h(\tilde{a})=\frac{\tilde{g}(0,1, \ldots, 1, \dot{1})}{\tilde{g}(1, \ldots, 1,0)}=E\left(X_{k+\Delta} / X_{k}=0\right)
$$

Using Equations 9 and 12 we finally obtain

$$
E X=\frac{h(\bar{a})}{2-f(\tilde{a})}+\frac{E\left[A^{2}\right]-2 \cdot E^{2}[A]+E[A]}{2(1-E A)}
$$

Wasted reservations: The bandwidth allocation in slot $k$ is wasted if $X_{k}=0$ and $X_{k-\Delta}>0$. Let $U_{k}=1$ if $X_{k-\Delta}>0$ and $X_{k}=0$, and $U_{k}=0$ otherwise. It follows that $E U=\operatorname{Prob}\left(X_{k}=0\right)-\operatorname{Prob}\left(X_{k-\Delta}=0, X_{k}=0\right)$. From (8) and (9) it follows that

$$
E U=(1-E A)\left(1-\frac{1}{2-f(\bar{a})}\right)=(1-E A)-\Pi_{0}
$$

For obtaining the values of $f(\tilde{a})$ and $h(\tilde{a})$ we need $\tilde{g}\left(u_{1}, \ldots, u_{\Delta}, \mathbf{i}\right), \tilde{g}\left(u_{1}, \ldots, u_{\Delta-1}, 0, u_{\Delta+1}\right), \tilde{g}\left(u_{1}, \ldots, u_{\Delta}, 0\right)$, $\tilde{g}\left(u_{1}, \ldots, u_{\Delta}, \dot{0}\right), \tilde{g}\left(u_{1}, \ldots, u_{\Delta}, \ddot{0}\right) \ldots, \ldots$, etc. These quantities are obtained by appropriately substituting values 0 and 1 in the arguments of Equation 6; the resulting equations are then used to obtain $f(\tilde{a})$ and $h(\tilde{a})$. Some of the necessary equations are derived in [7], and a general procedure is suggested. It is, however, difficult to carry out the procedure for large $\Delta$. Here we provide the explicit results for some small values of $\Delta$.

\section{B.1 Single Queue, $c=1, \Delta=1$}

We start with Equation 6.

$$
\begin{aligned}
& \tilde{g}\left(u_{1}, u_{2}\right)= \\
& \quad \tilde{a}\left(u_{2}\right) \cdot\left\{\frac{\tilde{g}\left(1, u_{1} \cdot u_{2}\right)}{u_{2}}+\left(1-\frac{1}{u_{2}}\right) \cdot\left[\tilde{g}\left(0, u_{1} \cdot u_{2}\right)\right.\right. \\
& \quad+\tilde{g}(1,0)-\tilde{g}(0,0)]\}
\end{aligned}
$$

Substituting $\left(u_{1}, u_{2}\right)=(0,0)$ in the above equation

$$
f(\tilde{a})=\frac{\tilde{g}(0,0)}{\tilde{g}(1,0)}=\tilde{a}(0)=a_{0}
$$

It follows the $2^{\text {nd }}$ order Markov chain is positive recurrent for $E A<1$ and $\operatorname{Pr}(X=0)=\frac{1-E A}{2-a_{0}}$. Substituting $u_{1}=0$, we get $\tilde{g}\left(0, u_{2}\right)=\tilde{a}\left(u_{2}\right) \cdot \tilde{g}(1,0)$ and from this we get

$$
h(\tilde{a})=\frac{\tilde{g}(0, \dot{1})}{\tilde{g}(1,0)}=E[A]
$$

From Equation 13

$$
E X=\frac{E A}{2-a_{0}}+\frac{E\left[A^{2}\right]-2 \cdot E^{2}[A]+E[A]}{2(1-E A)}
$$


B.2 Single Queue $c=1, \Delta=2$

Again, starting with Equation 6 .

$$
\begin{aligned}
& \tilde{g}\left(u_{1}, u_{2}, u_{3}\right)= \\
& \quad \tilde{a}\left(u_{3}\right) \cdot\left\{( 1 - \frac { 1 } { u _ { 3 } } ) \left[\tilde{g}\left(1, u_{1}, 0\right)+\tilde{g}\left(0, u_{1}, u_{2} \cdot u_{3}\right)\right.\right. \\
& \left.\left.\quad-\tilde{g}\left(0, u_{1}, 0\right)\right]+\frac{\tilde{g}\left(1, u_{1}, u_{2} \cdot u_{3}\right)}{u_{3}}\right\}
\end{aligned}
$$

For this case, we find that (see [7] for details)

$$
\begin{aligned}
& f(\tilde{a})=a_{0} \cdot\left(a_{0}+a_{1}-a_{0} \cdot a_{1}\right) \\
& h(\tilde{a})=2 . E[A]+2 \cdot a_{0}-a_{0}^{2}-1
\end{aligned}
$$

$$
E X=\frac{2 . E[A]+2 \cdot a_{0}-a_{0}^{2}-1}{2-a_{0}\left(a_{0}+a_{1}-a_{0} \cdot a_{1}\right)}+\frac{E\left[A^{2}\right]-2 . E^{2}[A]+E[A]}{2(1-E A)}
$$

\section{Arbitrary $c$ and $\Delta$}

The case $c=1, \Delta=3$ has been worked out in [7]. The case $c>1$ with $\Delta \geq 1$ has also been studied in [7]; each case, however, has to be worked out separately, and no general procedure appears to be available for obtaining the equations that need to be solved for each case.

\section{Single Node: Simulation Results}
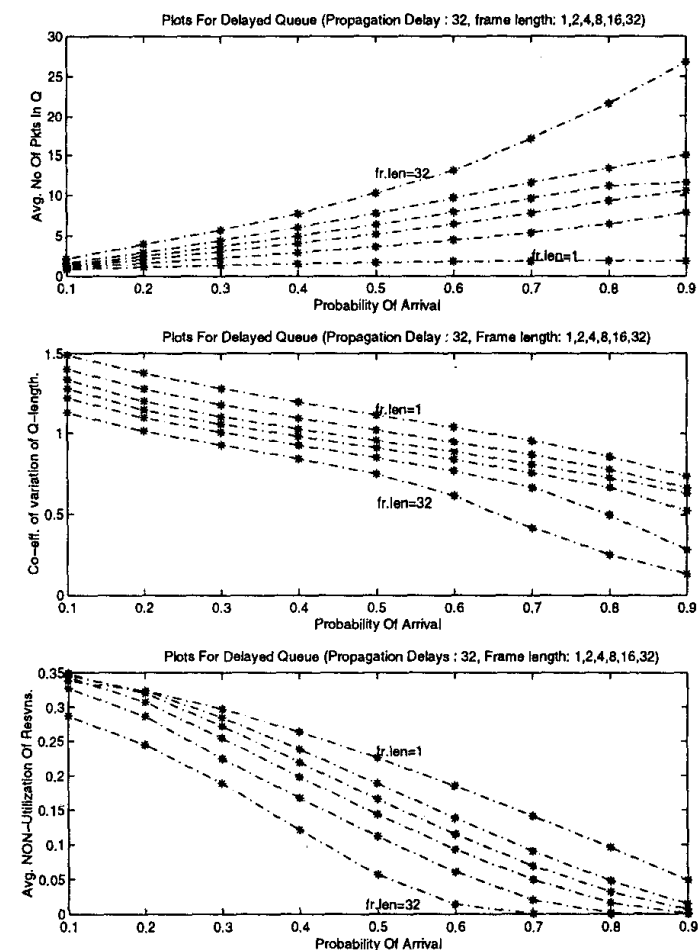

Fig. 2. Simulation results; single queue; Scheme 1; Bernoulli arrivals occur in the slots. $\Delta=32$ slots; frame length $=1,2,4,8,16,32$ slots.
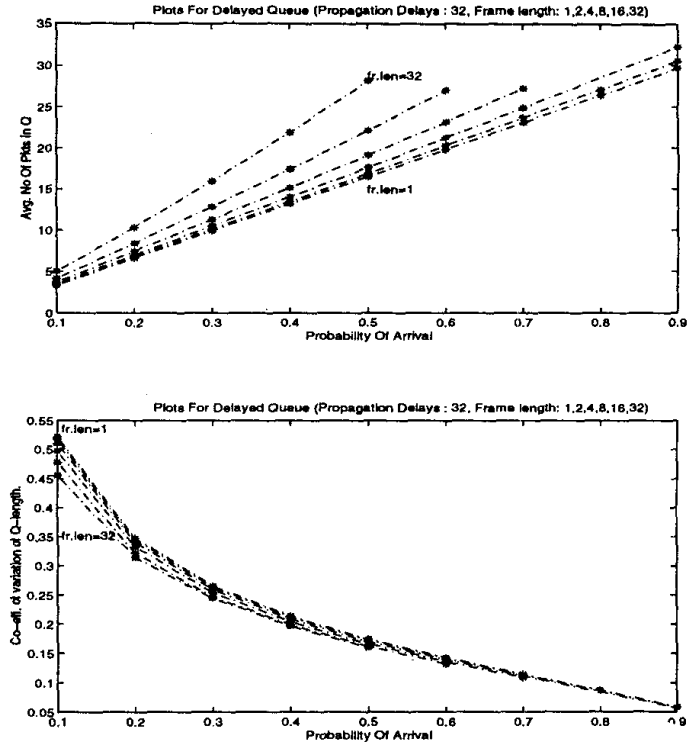

Fig. 3. Simulation results; single queue; Scheme 2; Bernoulli arrivals occur in the slots. $\Delta=32$ slots; frame length $=1,2,4,8,16,32$ slots.

In order to study a wider range of parameters than what the analysis can be evaluated for, we have used simulations. The system was simulated for a fixed $\Delta$ (in slots, i.e., the link speed and propagation delay are fixed), for Bernoulli arrivals, and varying frame lengths (i.e., $c$ ), for the reservation schemes Scheme 1 and Scheme 2. The objective here is to see the variation of the expected queue length and the co-efficient of variation $\left(\sqrt{\frac{E\left[X^{2}\right]}{(E[X])^{2}}-1}\right)$, for fixed propagation delay, and varying frame lengths and arrival probabilities for the two schemes. These results are interesting since reservation requests are only made at frame boundaries; shorter frames imply that the scheduler gets feedback more often and reservations are made more often. In the simulation model the arrival epochs are embedded at slot boundaries.

In Figures 2 and 3, we provide simulation results for $\Delta=32$, and $c=1,2,4,8,16,32$. We observe that for both schemes average queue length increases with increasing frame length. This is to be expected since with increasing frame length, packets queue up for longer to get service, and the service allocation is more bursty. For a given arrival rate the queue lengths are much higher for Scheme 2; this is because, by only allocating bandwidth when the scheduler is sure that the queue is not empty, often bandwidth is not allocated even when there are packets waiting. Notice that, in Scheme 1 , where there is a possibility of bandwidth wastage, the average nonutilised bandwidth decreases with increasing frame length; with large frame lengths, there is greater probability that if the past queue length is large then the bandwidth alloted will not be wasted. The coefficient of variation of the stationary queue length decreases with in- 
creasing arrival rate, and with increasing frame length; the decrease with frame length may partially be explained by the fact that the coefficient of variation of the arrival process over the frame length decreases with the frame length.

\section{Two Nodes: Simulation Results}

In this section, we explore, via simulation, a two node model as a representative of the multiple nodes problem, and assume Bernoulli arrivals to each node. Each frame consists of a single slot (i.e., $\mathrm{c}=1$ ). The arrival rates to the two nodes are taken to be equal. The slot in each frame is alloted to the longer of the two effective queue lengths as per Scheme 1 or Scheme 2.
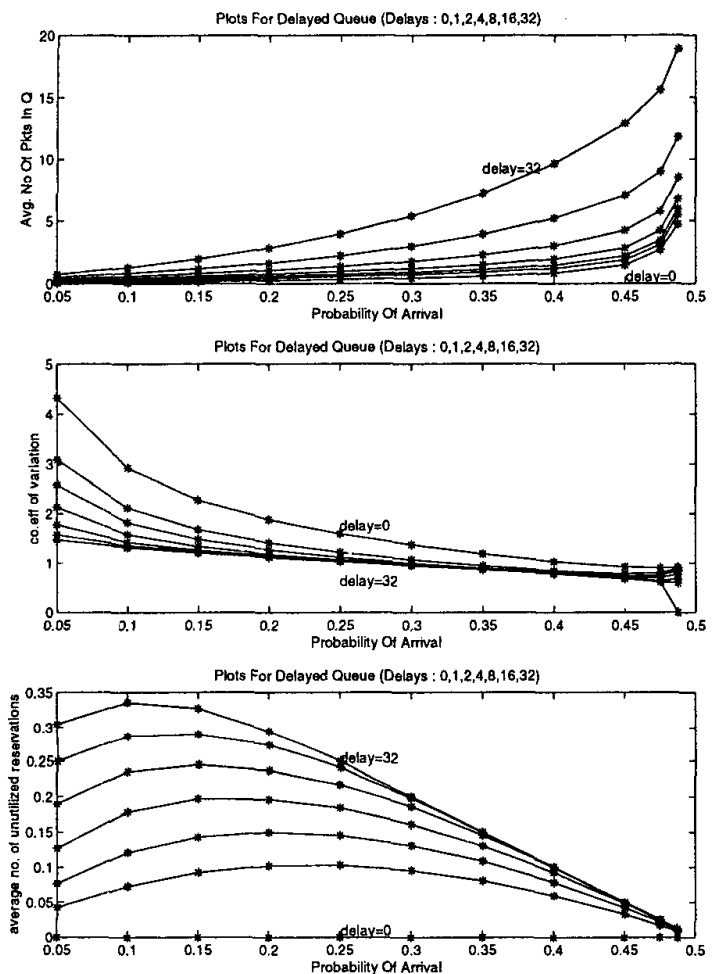

Fig. 4. Simulation results; two queues; Scheme 1; results are shown for a queue; equal rate Bernoulli arrivals to each queue; for various values of $\Delta$.

Figures 4 and 5 show the simulation results with two nodes for Scheme 1 and Scheme 2 respectively. The following observations are evident: for a given arrival rate, the mean queue length increases with increasing $\Delta$, since the "vacations" taken by the server are longer; the mean queue length is larger for Scheme 2; the coefficient of variation of the queue length decreases with increasing $\Delta$, and with increasing arrival rate; for Scheme 1 , the average wasted bandwidth increases with increasing $\Delta$; the wasted bandwidth first increases and then decreases with increasing arrival rate. Comparing with Figure 2, it can be inferred that if $\Delta$ is fixed, then the wasted bandwidth will decrease with increasing frame length. Observe that in the two node case
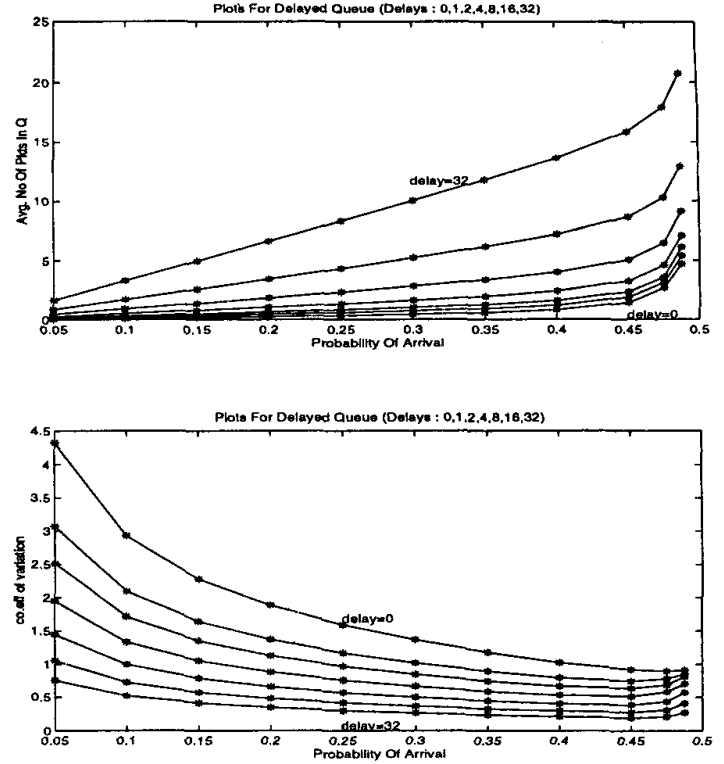

Fig. 5. Simulation results; two queues; Scheme 2; results are shown for a queue; equal rate Bernoulli arrivals to each queue; for various values of $\Delta$.

as the arrival rate approaches the stability limit ( 0.5 for each of the two queues, and 1 for a single queue) the mean queue length tends to infinity, whereas in the single node case the mean queue length tends to a finite value. The reason for this is that with $c=1$ and a single queue as the arrival rate approaches 1 a service is guaranteed at each slot, and there is at most one arrival in each slot; in the two queue case, there is uncertainty in the service allocation to each queue as only half the service effort is alloted to each queue, on the average.

\section{REFERENCES}

[1] Eitan Altman, Daniel Kofman and Uri Yechiali., "Discrete Time Queues With Delayed Information," Queveing Systems 19, pp. 361-376, 1995.

[2] Andrea Baiocchi, Marco Carosi, Marco Listanti and Aldo Roveri "Modeling of a Distributed Access Protocol for an ATM Satellite System: An Algorithmic Approach" IEEE JSAC Vol. 9, No.1, January $1991 \mathrm{pp} 65-75$.

[3] N. Celandroni and E. Ferro, "The FODA-TDMA Satellite Access Scheme: Presentation, Study of System, and Results," IEEE Trans. in Commn., Vol. COM-39, No. 12, December 1991.

[4] Supratim Deb, Performance Analysis of Reservation Multiple Access with Large Propagation Delay, Master of Engg Thesis, ECE Dept, Indian Institute of Science, Bangalore January 1999.

[5] Stephen F.W. Ng, Jon W. Mark, "A Multi access Model for Packet Switching with a Satellite having some processing capability" IEEE Trans. on Communication, Vol. COM-25, No. 1, January $1997 \mathrm{pp} 128-135$

[6] Fouad A. Tobagi, "Multiaccess Protocols in Packet Communication Systems," IEEE Trans. Comm., Vol. COM-28, No. 4, April 1980.

[7] S. Gouri Sankar, A Study Of The Effect Of Information Delays In Reservation Based Bandwidth Access, Master of Engg Thesis, ECE Dept., Indian Inst. of Science, Bangalore, June 1998.

[8] Jeffrey E. Wieselthier and Anthony Ephremides "A New Class of Protocols for Multiple Access in Satellite Networks" IEEE Trans. on Automatic Control, Vol. AC-25, No.5,October 1980 pp 865-879. 